\title{
Chirality oscillation of primordial gravitational waves during inflation
}

\author{
Yong Cai, ${ }^{a}$ Yu-Tong Wang ${ }^{a}$ and Yun-Song Piao ${ }^{a, b}$ \\ ${ }^{a}$ School of Physics, University of Chinese Academy of Sciences, \\ Beijing 100049, China \\ ${ }^{b}$ Institute of Theoretical Physics, Chinese Academy of Sciences, \\ P.O. Box 2735, Beijing 100190, China \\ E-mail: caiyong13@mails.ucas.ac.cn, wangyutong12@mails.ucas.ac.cn, \\ yspiao@ucas.ac.cn
}

ABStract: We show that if the gravitational Chern-Simons term couples to a massive scalar field $(m>H)$, the primordial gravitational waves (GWs) will show itself the chirality oscillation, i.e., the amplitudes of the left- and right-handed GWs modes will convert into each other and oscillate in their propagations. This oscillation will eventually develop a permanent difference of the amplitudes of both modes, which leads to nearly opposite oscillating shapes in the power spectra of the left- and right-handed primordial GWs. We discuss its implication to the CMB B-mode polarization.

KeYwords: Cosmology of Theories beyond the SM, String Field Theory

ARXIV EPRINT: 1608.06508 


\section{Contents}

1 Introduction 1

2 Chirality oscillation 2

3 The model 3

3.1 Numerical results 3

$\begin{array}{ll}3.2 & \text { Analytic estimation }\end{array}$

4 Discussion $\quad 9$

\section{Introduction}

General relativity (GR) is parity-free. However, in particle physics and string theory, the anomaly cancellation motivates the gravitational Chern-Simons (gCS) term [1, 2] (see [3] for the review), which is the leading-order parity-violating correction to GR (see also $[4,5]$ for the Weyl tensor). Thus the chirality of gravity might be a universal property of the UV-complete theory (see also $[6,7]$ ).

The primordial GWs $[8,9]$, predicted by inflation, may induce the B-mode polarization in the cosmological microwave background (CMB), and carry rich information about the early universe and the UV-complete gravity theory, e.g., [10-12]. During inflation, the primordial GWs generally originate from the vacuum fluctuation of the metric. The parityviolation of gravity induces chiral GWs [13], i.e., the left- and right-handed GWs modes propagate with different behaviors. However, in models of inflation in which a pseudoscalar field interacts with the gauge field [14-19] (see also [20]), the gauge field is also the source of parity-violating GWs. These two sources seem undistinguishable.

During inflation, the running of the propagating speed of GWs will result in oscillating shapes $[10,11,21]$ or a blue tilt $[22]$ in the GWs spectrum. Similarly, the chirality will also be manifested in the primordial GWs spectrum [13, 23-26], which may bring the TB/EBmode correlation in CMB [27-29]. Due to the importance of the parity-violating correction of gravity for the particle physics and string theory, it is significant to search for its unique observable fingerprint.

In this paper, we show that if the gCS term couples to a massive scalar field $(m>H)$, the primordial GWs will show itself the chirality oscillation, i.e., the amplitudes of left- and right-handed GWs modes will convert into each other and oscillate in their propagations. This is a novel phenomenon of the chirality of gravity, which has not been uncovered before. We illustrate the implications of the chirality oscillation to the primordial GWs spectrum, and its imprints in the CMB B-mode polarization. 


\section{Chirality oscillation}

We begin with the quadratic action for the tensor perturbation

$$
S_{\gamma \gamma}=\int d \tau d^{3} x\left[\frac{M_{p}^{2} a^{2}}{8}\left(\gamma_{i j}^{\prime 2}-\left(\vec{\nabla} \gamma_{i j}\right)^{2}\right)+\mathcal{L}_{H D}\right],
$$

where $\tau=\int d t / a$ and ${ }^{\prime}=d / d \tau, M_{p}$ is the Planck scale and $\mathcal{L}_{H D}$ is the higher-order derivative corrections with the parity violation. Considering only the leading order terms, we could write

$$
\mathcal{L}_{H D}=-\frac{M_{p}^{2}}{8}\left[\frac{\xi_{1}}{\mathcal{M}} \epsilon^{i j k}\left(\partial_{i} \gamma_{j l}\right)^{\prime}\left(\gamma_{k}^{l}\right)^{\prime}+\frac{\xi_{2}}{\mathcal{M}} \epsilon^{i j k} \partial_{i} \partial_{l} \gamma_{j q} \partial^{l} \gamma_{k}^{q}\right]
$$

where $\epsilon^{i j k}$ is the Levi-Cevita symbol, $\xi_{1}$ and $\xi_{2}$ are time-dependent parameters, and $\mathcal{M}$ is the cutoff scale. The gCS term corresponds to $\xi_{1}=-\xi_{2}$ [13, 23, 30], while $W \widetilde{W}$ corresponds to $\xi_{1}=0[4,5,12]$, with $W$ being the Weyl tensor.

In the Fourier space, we have

$$
\gamma_{i j}(\tau, \mathbf{x})=\sum_{s=L, R} \int \frac{d^{3} \mathbf{k}}{(2 \pi)^{3}} \gamma_{\mathbf{k}}^{(s)}(\tau) p_{i j}^{(s)}(\mathbf{k}) e^{i \mathbf{k} \cdot \mathbf{x}},
$$

where the polarization tensors $p_{i j}^{(s)}$ satisfy $p_{i j}^{(R)} p^{i j(R)}=p_{i j}^{(L)} p^{i j(L)}=0, p_{i j}^{(R)} p^{i j(L)}=2$ and $i k_{l} \epsilon^{n l j} p_{i j}{ }^{(s)}=k \lambda^{(s)} p_{i}^{n(s)}$. The parameter $\lambda^{(L)}=-1$ and $\lambda^{(R)}=1$ correspond to the leftand the right-handed mode, respectively.

The equation of motion of $\gamma_{\mathbf{k}}^{(s)}$ is

$$
u_{\mathbf{k}}^{(s)^{\prime \prime}}+\left[\left(c_{T k}^{(s)}\right)^{2} k^{2}-\frac{z^{(s)^{\prime \prime}}}{z^{(s)}}\right] u_{\mathbf{k}}^{(s)}=0
$$

where $u_{\mathbf{k}}^{(s)}=z^{(s)} \gamma_{\mathbf{k}}^{(s)}$ and $z^{(s)}=\frac{a}{2} \sqrt{1-\lambda^{(s)} \frac{k}{a^{2}} \frac{\xi_{1}}{\mathcal{M}}}$, and the effective sound speed of different polarization modes

$$
c_{T k}^{(s)}=\left(1+\lambda^{(s)} \frac{k}{a^{2}} \frac{\xi_{2}}{\mathcal{M}}\right)^{1 / 2}\left(1-\lambda^{(s)} \frac{k}{a^{2}} \frac{\xi_{1}}{\mathcal{M}}\right)^{-1 / 2} .
$$

Here, $\xi_{1}<a$ is required, otherwise the ghost modes will appear at the cutoff scale $k / a=\mathcal{M}$, see [31].

Initially, the perturbations are deep inside their horizon, i.e., $\left(c_{T k}^{(s)}\right)^{2} k^{2} \gg z^{(s)^{\prime \prime}} / z^{(s)}$, thus $u_{\mathbf{k}}^{(s)} \simeq e^{i c_{T k}^{(s)} k \tau} / \sqrt{2 c_{T k}^{(s)} k}$. The power spectrum of primordial GWs is

$$
P_{T}^{(s)}=\frac{k^{3}}{2 \pi^{2}}\left|\gamma_{\mathbf{k}}^{(s)}\right|^{2}, \quad a H / c_{T k}^{(s)} k \gg 1 .
$$

The chiral parameter $\Delta \chi=\left(P_{T}^{(L)}-P_{T}^{(R)}\right) /\left(P_{T}^{(L)}+P_{T}^{(R)}\right)$ reflects the intensity of parity violation of primordial GWs. 
When $\xi_{1}$ or $\xi_{2} \neq 0, \gamma_{\mathbf{k}}^{(s)}$ with different polarizations will have different evolutions, the primordial GWs are chiral. In sting theory, $\xi_{1}$ and $\xi_{2}$ actually are moduli-dependent, see [3], and the massive moduli fields $(m>H)$ are ubiquitous. The nontrivial evolution (especially the oscillation) of the moduli field indicates the nontrivial variations of $\xi_{1}$ and $\xi_{2}$, which may induce oscillation of the chiral term $c_{T k}^{(s)}$ or $z^{(s)^{\prime \prime}} / z^{(s)}$ in eq. (2.4). It is this physics that induces the chirality oscillation of the primordial GWs, similar to the case in refs. $[10,11,21]$ where the nontrivial variation (such as oscillation) of the propagating speed of GWs induces oscillations in the GWs modes as well as in the GWs power spectrum.

Generally, the single field slow-roll inflation is thought as the effective model after all massive modes are integrated out. However, during inflation, the massive fields could be excited and oscillate around its minimum. This will inevitably induce the chirality oscillation of the primordial GWs, which could be encoded in the power spectrum of primordial GWs, as will be showed.

\section{The model}

We will illustrate the idea of the chirality oscillations with a workable model in detail, in which the background is set as the inflation with $\epsilon=-\dot{H} / H^{2} \ll 1$, and $\phi$ is a massive modulus field with

$$
S_{\phi}=\frac{M_{p}^{2}}{2} \int d^{4} x \sqrt{-g}\left(-\partial_{\mu} \phi \partial^{\mu} \phi-2 V(\phi)+\frac{f(\phi)}{4 M_{p}^{2}} R \wedge R\right),
$$

where $R \wedge R=\epsilon^{\alpha \beta \gamma \delta} R_{\alpha \beta \mu \nu} R_{\gamma \delta}^{\mu \nu}$ is the gCS term. Thus we have $\xi_{1}=-\xi_{2}=\frac{\mathcal{M} f^{\prime}}{M_{p}^{2}}$ in eq. (2.2), so

$$
z^{(s)}=\frac{a}{2} \sqrt{1-\lambda^{(s)} \frac{k}{a^{2}} \frac{f^{\prime}}{M_{p}^{2}}}
$$

and $c_{T k}^{(s)}=1$. In addition, it is required that $\frac{1}{2} \dot{\phi}^{2}+V(\phi) \ll 3 H^{2}$, and $\left|-\frac{1}{2} \dot{\phi}^{2}\right| \ll|\dot{H}|$, so that the background is unaffected by $\phi$.

In this model, if $\phi$ oscillates around the minimum of its potential during inflation, the chiral term $z^{(s)^{\prime \prime}} / z^{(s)}$ in eq. (2.4) could experience nontrivial oscillation, which will induce the chirality oscillation of primordial GWs, as explained in section 2. Here, we would like to point out that the oscillation of $\phi$ around the minimum of $V(\phi)$ is a sufficient condition rather than a necessary one for producing the chirality oscillation of GWs as long as $f$ is $\phi$-dependent, since what plays the key role is $z^{(s)^{\prime \prime}} / z^{(s)}$, which depends also on the shape of $f(\phi)$. For instance, a trivially evolved $\phi$ may also generate the chirality oscillation of GWs if $f(\phi)$ is delicately designed. However, in the following, we will focus on the case where it is the oscillation of $\phi$ that generates the chirality oscillation of primordial GWs.

\subsection{Numerical results}

Below, we will numerically show the evolutions of the left- and right-handed GWs modes and the corresponding spectrum. We define $\alpha=\ln \left(a / a_{0}\right)$, where there may be a difference 
of a constant between $\alpha$ and the usually used e-folding number $N$. Then, eq. (2.4) can be rewritten as

$$
u_{\mathbf{k}, \alpha \alpha}^{(s)}+\left(1+\frac{H_{, \alpha}}{H}\right) u_{\mathbf{k}, \alpha}^{(s)}+\frac{1}{a^{2} H^{2}}\left(k^{2}-\frac{z^{(s)^{\prime \prime}}}{z^{(s)}}\right) u_{\mathbf{k}}^{(s)}=0,
$$

where $z^{(s)}=\frac{a}{2} \sqrt{1-\lambda^{(s)} \frac{k H}{a} \frac{f, \alpha}{M_{p}^{2}}}$ and the subscript ", $\alpha$ " is the derivative with respect to $\alpha$, and the Hubble parameter is written as $H(\alpha)=H_{0} e^{-\epsilon \alpha}$ with $H_{0}$ set by the amplitude of curvature perturbation.

Generally, $f(\phi) \sim \phi$, see [3] for a review. To avoid the ghost mode, we require that the parity-violation only occur in the region around $\phi=0$. Thus we set

$$
f(\phi)=\frac{A_{f} \phi}{\phi^{2}+c}
$$

where $A_{f}$ and $c$ are dimensionless, which gives $f_{, \alpha} \approx g \phi_{, \alpha}$ for $\phi \rightarrow 0$, in which $g=A_{f} / c$ is the coupling coefficient of $\phi$ to the gCS term, and $f_{, \alpha} \approx 0$ for $|\phi| \gg \sqrt{c}$. During inflation, the massive modulus field might be excited and then relax towards its local minimum, and oscillate rapidly around it. Thus for the simulation, we set

$$
V(\phi)=\frac{m^{2} \phi^{2} / 2}{1+\left(\phi M_{p} / \Lambda\right)^{2}},
$$

where $\Lambda$ has the mass dimension. When $|\phi| \gg \Lambda / M_{p}$,

$$
V(\phi) M_{p}^{2} \simeq m^{2} \Lambda^{2} \ll \rho_{\text {inf }}=3 H^{2} M_{p}^{2}
$$

is constant, which insures that the inflation background is unaffected by $\phi$. Additionally, the initial condition of $\phi$ is set by $\phi\left(\alpha_{\text {ini }}\right)=\phi_{\text {ini }}$ and $\phi_{, \alpha}\left(\alpha_{\text {ini }}\right)=0$.

Note that, since in our model it is the oscillation of $\phi$ that induces the chirality oscillation of primordial GWs, any potential with a minimum could do the job. Additionally, the chirality oscillation would present for any $f(\phi) \sim \phi$ when $\phi$ is small. To demonstrate this point, we will also show the oscillation of $\Delta \chi$ with $f(\phi)=A_{f} \phi /(\phi+c)$. Although the shape of the chirality oscillation could be a little model-dependent (which should be attributed to the transition of $f(\phi)$ around $\phi= \pm \sqrt{c}$ or $\pm c$ ), the phenomenon of chirality oscillation of primordial GWs is quite general.

We plot the evolutions of $\phi, \xi_{1}$ (i.e., $\left.f^{\prime}\right)$ and $z^{(s)^{\prime \prime}} / z^{(s)}$ in figure 1 , and plot $\left|u_{\mathbf{k}}^{(s)}\right|$ and $\left|\gamma_{\mathbf{k}}^{(s)}\right|$ in figure 2 for the mode $k=10^{-3} \mathrm{Mpc}^{-1}$, which is at the CMB scale and whose corresponding frequency is about $10^{-17} \mathrm{~Hz}$. The chirality oscillations of the primordial GWs modes start at around $\alpha=8$, since $\phi$ starts oscillating and $z^{(s)^{\prime \prime}} / z^{(s)}$ starts its nontrivial oscillation at that time. We see that the chirality oscillation of primordial GWs is encoded in its power spectrum, as was showed in figure 3(a), see also [32] for the GWsgauge field oscillation. The amplitudes of the left- and right-handed GWs mode oscillate almost (but not exactly) symmetrically, and will eventually arrive at different values. This difference with respect to the comoving wave number is reflected in the oscillation of $\Delta \chi$, see figures 3(c) and 3(d). It is also noticed that the broken symmetry of the evolutions of left- and right-handed GWs modes, i.e., $\left|\gamma^{(L)}\right|+\left|\gamma^{(R)}\right| \neq 2\left|\gamma^{(0)}\right|$, actually also imprint an oscillating fingerprint in $P_{T}=P_{T}^{(L)}+P_{T}^{(R)}$, as was showed in figure $3(\mathrm{~b})$. 


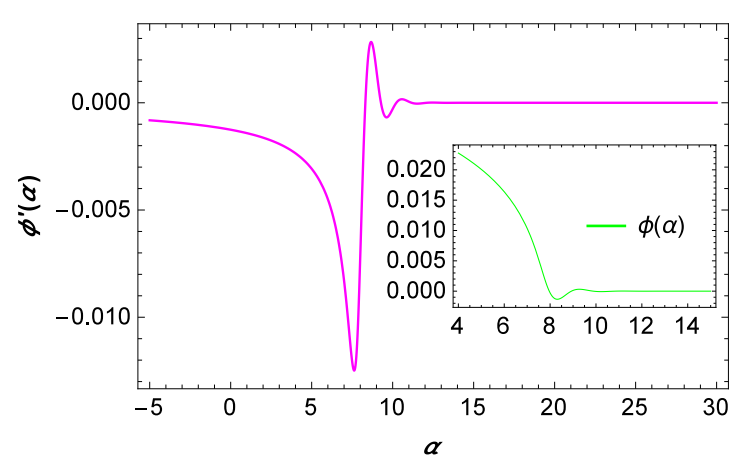

(a) $\phi(\alpha)$ and $\phi, \alpha$

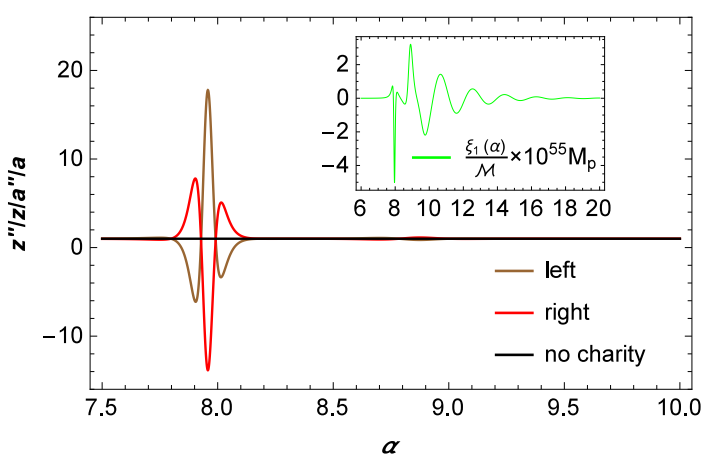

(b) $\frac{z^{(s) \prime \prime} / z^{(s)}}{a^{\prime \prime} / a}$ and $\xi_{1}$

Figure 1. Evolution of $\phi, \xi_{1}$ and $z^{(s)^{\prime \prime}} / z^{(s)}$ when $\alpha_{\text {ini }}=-22.8, \phi_{\text {ini }}=0.044, \epsilon=0.003, a_{0}=10^{-60}$, $H_{0}=2.72 \times 10^{-5} M_{p}, m=9.6 \times 10^{-5} M_{p}, \Lambda=0.01 M_{p}, A_{f}=325$, and $c=4 \times 10^{-7}$. In (b), "left", "right" and "no chirality" stand for $\lambda^{(s)}=-1,1$ and 0 , respectively.

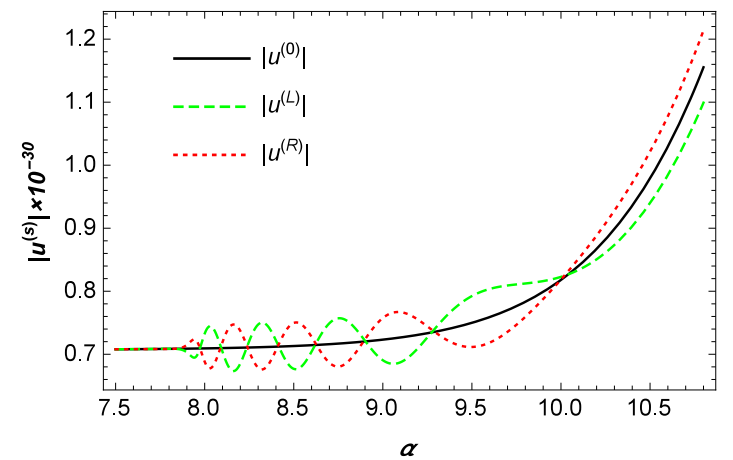

(a) $\left|u^{(s)}\right|$

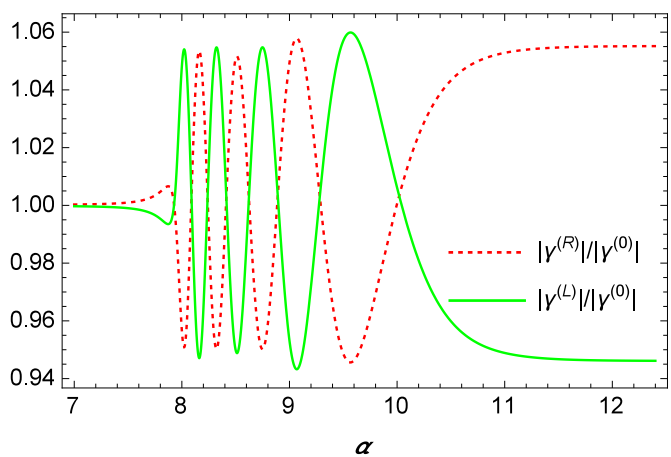

(b) $\left|\gamma^{(s)}\right| /\left|\gamma^{(0)}\right|$

Figure 2. Chirality oscillation of primordial GWs modes. We set $k=10^{-3} \mathrm{Mpc}^{-1}, \alpha_{\text {ini }}=-22.8$, $\phi_{\text {ini }}=0.044, \epsilon=0.003, a_{0}=10^{-60}, H_{0}=2.72 \times 10^{-5} M_{p}, m=9.6 \times 10^{-5} M_{p}, \Lambda=0.01 M_{p}$, $A_{f}=325$, and $c=4 \times 10^{-7}$. "(L)", "(R)" and "(0)" stand for $\lambda^{(s)}=-1,1$ and 0 , respectively.

The chiral GWs will induce non-vanishing TB/EB-mode correlation at CMB last scatting surface, $C_{l}^{T / E, B} \sim \int \frac{d k}{k} \Delta \chi P_{T}\left[\Delta_{l}^{T / E}(k) \Delta_{l}^{B}(k)\right]$ (e.g., $[27,28]$ ), if the modulus field happens to oscillate at the time of $\sim 60$ efolds before the end of inflation. ${ }^{1}$ For parameters used in the numerical calculation, the perturbation mode with $k \sim 10^{-4} \mathrm{Mpc}^{-1}$ exits horizon at about $\alpha=8$, where $k \sim a H$ is used for the estimation. Thus after $a_{0}$ and $H_{0}$ are fixed, there is some fine-tuning of the parameters $\phi_{\text {ini }}$ and $\alpha_{\text {ini }}$, which set the initial condition of $\phi$, so that $\phi$ starts oscillating at about $\alpha=8$. We plot the TB/EB-mode spectrum in figure 4. We see that the chirality oscillation of primordial GWs will bring obvious wiggles in the TB/EB-mode spectrum, which is a novel phenomenon has not been uncovered before.

\footnotetext{
${ }^{1}$ The perturbation mode with comoving wave number $k \sim 10^{-4} \mathrm{Mpc}^{-1}$ exits horizon at about the time of $\sim 60$ efolds before the end of inflation.
} 


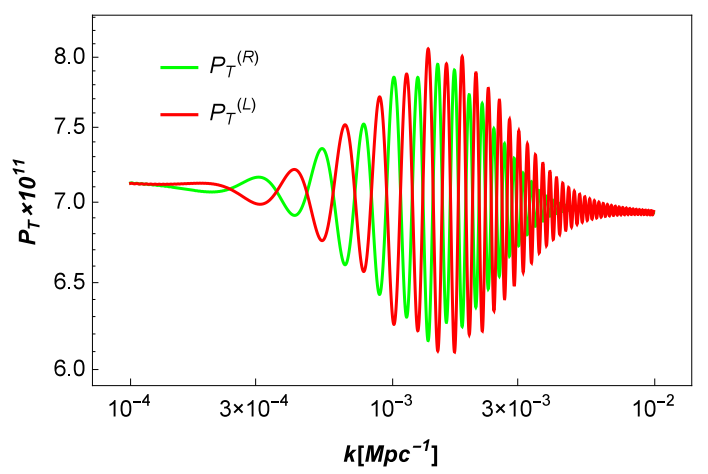

(a) $\quad P_{T}^{(s)}$ for $f(\phi)=\frac{A_{f} \phi}{\phi^{2}+c}$

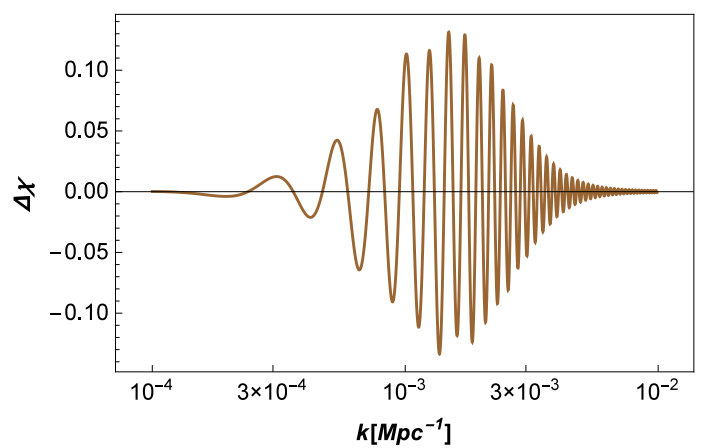

(c) $\Delta \chi$ for $f(\phi)=\frac{A_{f} \phi}{\phi^{2}+c}$

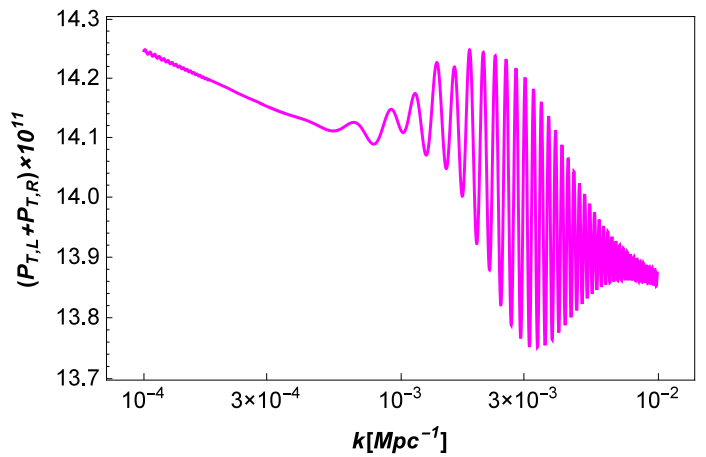

(b) $P_{T}^{(L)}+P_{T}^{(R)}$ for $f(\phi)=\frac{A_{f} \phi}{\phi^{2}+c}$

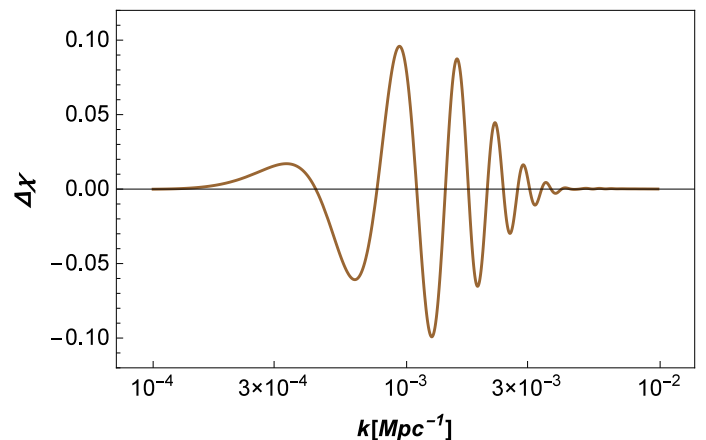

(d) $\Delta \chi$ for $f(\phi)=\frac{A_{f} \phi}{\phi+c}$

Figure 3. Parity violating power spectrum of primordial GWs. We set $\alpha_{\text {ini }}=-22.8, \phi_{\text {ini }}=0.044$, $\epsilon=0.003, a_{0}=10^{-60}, H_{0}=2.72 \times 10^{-5} M_{p}, m=9.6 \times 10^{-5} M_{p}$ and $\Lambda=0.01 M_{p}$. Note that $A_{f}=325$ and $c=4 \times 10^{-7}$ for (a)(b)(c) while $A_{f}=1.625 \times 10^{6}$ and $c=2 \times 10^{-3}$ for (d). "(L)" and "(R)" stand for $\lambda^{(s)}=-1$ and 1 , respectively. We have set the cutoff scale at $\mathcal{M} \sim 10^{-4} M_{p}$, which corresponds to $k_{\text {cut }} \sim a \mathcal{M} \sim 10^{-2} \mathrm{Mpc}^{-1}$ while $\alpha \approx 12$.

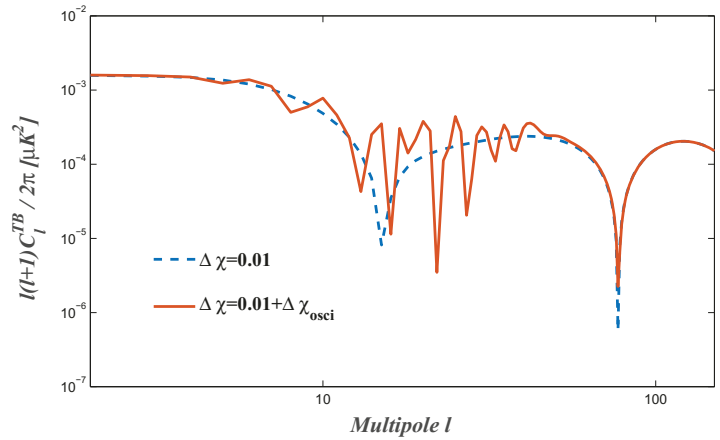

(a) $T B$

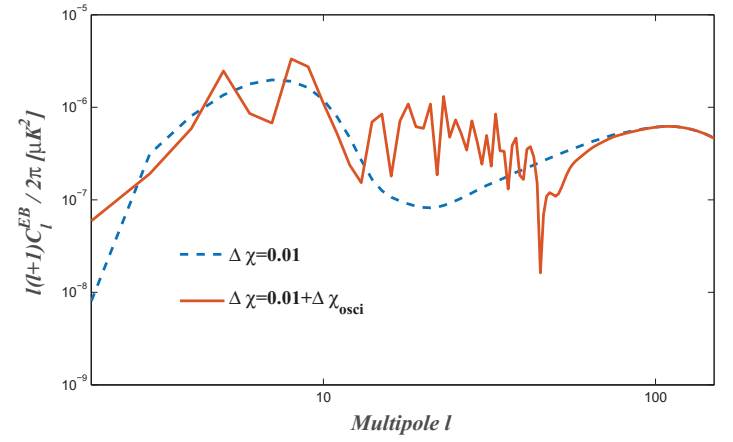

(b) $E B$

Figure 4. The CMB TB/EB spectrum obtained by modifying the CAMB code. Here, $\Delta \chi=0.01$ is used as a reference to highlight the observational feature of chirality oscillation, and $\Delta \chi_{o s c i}$ actually equals the $\Delta \chi$ given by figure $3(\mathrm{c})$. 


\subsection{Analytic estimation}

With (3.2) and the numerical calculation, we approximately have

$$
\frac{z^{(s)^{\prime \prime}}}{z^{(s)}} \approx a^{2} H^{2}\left(2-\frac{\lambda^{(s)} k H}{2 a M_{p}^{2}} f_{, \alpha \alpha \alpha}\right) .
$$

Then noting $a H \approx-1 / \tau$, eq. (2.4) can be approximated as

$$
u_{\mathbf{k}}^{(s)}+\left[k^{2}-\frac{\lambda^{(s)} A_{*} k}{2 \tau}-\frac{2}{\tau^{2}}\right] u_{\mathbf{k}}^{(s)}=0,
$$

where $A_{*}=H^{2} f_{, \alpha \alpha \alpha} / M_{p}^{2}$ is dimensionless, which reflects the effect of parity-violating correction of gravity.

Without loss of generality, we assume that before $\tau=\tau_{\text {mat }}, A_{*}=$ constant, and hereafter $A_{*}=0$, where $\tau_{\text {mat }}$ is the comoving time at the matching surface and depends on $\phi_{\text {ini }}$ and $\alpha_{\text {ini }}$ in the model. Thus when $\tau<\tau_{\text {mat }}$, the solution is

$$
u_{\mathbf{k} 1}^{(s)}=c_{11} \cdot M\left(\frac{i}{4} \lambda^{(s)} A_{*}, \frac{3}{2}, 2 i k \tau\right)+c_{12} \cdot W\left(\frac{i}{4} \lambda^{(s)} A_{*}, \frac{3}{2}, 2 i k \tau\right),
$$

where $M$ and $W$ are the Whittaker functions. Initially, the perturbations are deep inside the horizon, i.e., $-k \tau \gg 1$. Using

$$
W(x, y, z) \approx e^{-z / 2} z^{x}\left(1-\frac{1-4 x+4 x^{2}-4 y^{2}}{4 z}\right)+\mathcal{O}\left[\frac{1}{z^{2}}\right]
$$

for $|z| \gg 1$ and

$$
M(x, y, z)=\frac{\Gamma(2 y+1)}{\Gamma\left(\frac{1}{2}+x+y\right)} e^{-i \pi\left(\frac{1}{2}+y-x\right)} W(x, y, z)+\frac{\Gamma(2 y+1)}{\Gamma\left(\frac{1}{2}+y-x\right)} e^{i x \pi} W(-x, y,-z),
$$

we have

$$
c_{11}=0, \quad c_{12}=\frac{1}{\sqrt{2 k}} e^{-\frac{\lambda(s)}{8} A_{*} \pi},
$$

so that the initial state satisfies $u_{\mathrm{k}}^{(s)} \simeq \frac{1}{\sqrt{2 k}} e^{i k \tau}$.

When $\tau>\tau_{\text {mat }}$, the solution is

$$
u_{\mathbf{k} 2}^{(s)}=\frac{\sqrt{-\pi \tau}}{2}\left[c_{21} H_{3 / 2}^{(1)}(-k \tau)+c_{22} H_{3 / 2}^{(2)}(-k \tau)\right],
$$

where $H_{3 / 2}^{(1)}$ and $H_{3 / 2}^{(2)}$ are the $3 / 2$ th order Hankel functions of the first and second kinds, respectively. Thus we have

$$
P_{T}^{(s)}=P_{T, \text { inf }}^{(0)} g\left(k, \tau_{\text {mat }}, A_{*}, \lambda^{(s)}\right),
$$

where

$$
g\left(k, \tau_{\text {mat }}, A_{*}, \lambda^{(s)}\right)=\left|c_{21}-c_{22}\right|^{2},
$$




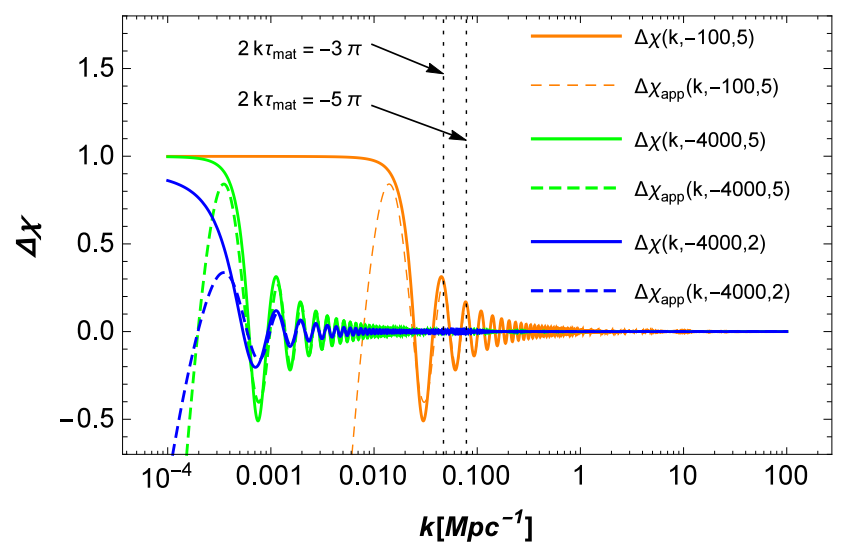

Figure 5. $\Delta \chi\left(k, \tau_{\mathrm{mat}}, A_{*}\right)$ is given by eqs. (3.15) to (3.17), and $\Delta \chi_{\text {app }}\left(k, \tau_{\mathrm{mat}}, A_{*}\right)$ is given by eq. (3.19). The first and the second peak of $\Delta \chi$ correspond to $2 k \tau_{\text {mat }}=-3 \pi$ and $-5 \pi$, respectively.

$P_{T, \text { inf }}^{(0)}=H^{2} / \pi^{2}$ and $\tau_{\text {mat }}$ corresponds to the match surface. Then, the chirality parameter

$$
\Delta \chi\left(k, \tau_{\text {mat }}, A_{*}\right)=\frac{g\left(k, \tau_{\text {mat }}, A_{*},-1\right)-g\left(k, \tau_{\text {mat }}, A_{*}, 1\right)}{g\left(k, \tau_{\text {mat }}, A_{*},-1\right)+g\left(k, \tau_{\text {mat }}, A_{*}, 1\right)} .
$$

By requiring the continuities of $u_{\mathbf{k}}^{(s)}$ and $u_{\mathbf{k}}^{(s)^{\prime}}$ at $\tau_{\text {mat }},{ }^{2}$ with the Tricomi confluent hypergeometric function $U(a, b, x)$, we obtain

$$
\begin{aligned}
c_{21}= & \frac{1}{2} e^{-\frac{\lambda^{(s)}}{8} A_{*} \pi}\left[\left(4-4 i k \tau_{\text {mat }}\right) U\left(1-\frac{i \lambda^{(s)} A_{*}}{4}, 4,2 i k \tau_{\text {mat }}\right)\right. \\
& \left.+\left(\lambda^{(s)} A_{*}\left(i+k \tau_{\text {mat }}\right)-4\right) U\left(2-\frac{i \lambda^{(s)} A_{*}}{4}, 4,2 i k \tau_{\text {mat }}\right)\right], \\
c_{22}= & \frac{i e^{-\frac{\lambda^{(s)}}{8} A_{*} \pi} \sqrt{\pi}}{8 \sqrt{-2 k \tau_{\text {mat }}}}\left[-4 H_{3 / 2}^{(1)}\left(-k \tau_{\text {mat }}\right) W\left(1+\frac{i}{4} \lambda^{(s)} A_{*}, \frac{3}{2}, 2 i k \tau_{\text {mat }}\right)\right. \\
& +\left(4 k \tau_{\text {mat }} H_{1 / 2}^{(1)}\left(-k \tau_{\text {mat }}\right)+\left(4-i \lambda^{(s)} A_{*}+4 i k \tau_{\text {mat }}\right) H_{3 / 2}^{(1)}\left(-k \tau_{\text {mat }}\right)\right) \\
& \left.\times W\left(\frac{i}{4} \lambda^{(s)} A_{*}, \frac{3}{2}, 2 i k \tau_{\text {mat }}\right)\right] .
\end{aligned}
$$

We plot $\Delta \chi\left(k, \tau_{\text {mat }}, A_{*}\right)$ in figure 5. The amplitude of the oscillation is determined by $A_{*}$, while the position of the oscillation is determined by $\tau_{\text {mat }}$. What we are interested in is the oscillation of $\Delta \chi$ when $k>-1 / \tau_{\text {mat }}$. Though the amplitude of the oscillation in figure 5 is slightly larger than that in figure $3(\mathrm{c})$, due to the oversimplified assumption we made, we may use the first or the second peak of $\Delta \chi\left(k, \tau_{\text {mat }}, A_{*}\right)$ in figure 5 , as the estimation of the overall oscillating amplitude in figure 3(c).

In the limit $-k \tau_{\text {mat }} \gg 1$, we have

$$
g\left(k, \tau_{\text {mat }}, A_{*}, \lambda^{(s)}\right)=1-\frac{\lambda^{(s)} A_{*}}{4 k \tau_{\text {mat }}} \cos \left(2 k \tau_{\text {mat }}\right)+\mathcal{O}\left[\frac{1}{\left(-k \tau_{\text {mat }}\right)^{2}}\right],
$$

\footnotetext{
${ }^{2}$ When both $a$ and $a^{\prime}$ are continuous at $\tau_{\text {mat }}$, the continuities of $u$ and $u^{\prime}$ are equivalent to the continuities of $\gamma_{\mathbf{k}}$ and $\gamma_{\mathbf{k}}^{\prime}$.
} 
thus

$$
\Delta \chi_{\mathrm{app}}\left(k, \tau_{\mathrm{mat}}, A_{*}\right) \approx \frac{A_{*}}{4 k \tau_{\mathrm{mat}}} \cos \left(2 k \tau_{\mathrm{mat}}\right)
$$

where eq. (3.10) and $U(x, y, z)=z^{-x}\left(1-\frac{x+x^{2}-x y}{z}\right)+\mathcal{O}\left[\frac{1}{z^{2}}\right]$ for $|z| \gg 1$ are used. Although eq. (3.19) is obtained for $-k \tau_{\text {mat }} \gg 1$, it is still able to mimic the oscillation part of $\Delta \chi$ pretty well, ${ }^{3}$ see figure 5 . From eq. (3.19) we can infer intuitively that the maximum value of the peaks in $\Delta \chi$ app and $\Delta \chi$ corresponds to $\cos \left(2 k \tau_{\text {mat }}\right)=-1$. Combined with the numerical results given in figure 5 , we find the first and the second peak of $\Delta \chi$ correspond to $2 k \tau_{\text {mat }}=-3 \pi$ and $-5 \pi$, respectively. The peak values of the first and the second peak of $\Delta \chi$ are $\frac{A_{*}}{6 \pi}$ and $\frac{A_{*}}{10 \pi}$, respectively. When the values of the parameters we used in figure $3(\mathrm{c})$ are substituted into eq. (3.19), we roughly have $\Delta \chi \sim 0.2$, which is consistent with our numerical result.

Additionally, the parameter $\tau_{\text {mat }}$ determines the wavenumber at which the oscillation peak appears, as we can see from figure 5 . However, since the main purpose of this analytical approximation is to have an estimation of the amplitude of the chirality oscillation, the value of $\tau_{\text {mat }}$ is not so important here.

As has been mentioned, $A_{*}=H^{2} f_{, \alpha \alpha \alpha} / M_{p}^{2}$, which approximately is $A_{*} \simeq \dddot{g} \ddot{\phi} / H M_{p}^{2}$. During the rapid oscillating of $\phi$, we neglect the effect of cosmological expansion, and have $|\dddot{\phi}| \simeq m^{2}|\dot{\phi}| \simeq m^{3} \Lambda / M_{p}$. Thus

$$
A_{*} \simeq \frac{g m^{3} \Lambda}{H M_{p}^{3}}<g \frac{m^{2}}{M_{p}^{2}}
$$

where (3.6) is used. This indicates that the intensity of the chirality oscillation is determined by the mass of scalar field and its coupling $g$ to the gCS term. Generally, the rapid oscillation requires $H<m \ll M_{p}$. Thus a larger $A_{*}$ implies a larger $g$. In string theory, $g=\pi^{2} \sqrt{g_{s} / 2} M_{p}^{2} / M_{s}^{2}$, e.g., $[3,23]$, where $M_{s}$ is the string scale and $g_{s}$ is the string coupling, we may have $g \gg 1$ for $M_{s} \ll M_{p}$. Thus the amplitude of oscillation in CMB TB/EB spectrum may be straightly linked to the stringy parameters.

\section{Discussion}

In summary, we found a novel phenomenon of the chirality of gravity at inflation scale, i.e., the amplitudes of left- and right-handed primordial GWs modes will convert into each other and oscillate in their propagations. We illustrated it by applying the gCS term coupling to a massive scalar field. ${ }^{4}$ This chirality oscillation will eventually develop a permanent

\footnotetext{
${ }^{3}$ Up to the next order,$$
\Delta \chi=\frac{4 A_{*}\left[2 k \tau_{\text {mat }} \cos \left(2 k \tau_{\text {mat }}\right)-3 \sin \left(2 k \tau_{\text {mat }}\right)\right]}{A_{*}^{2}+32 k^{2} \tau_{\text {mat }}^{2}-2 A_{*}^{2} \cos \left(2 k \tau_{\text {mat }}\right)}+\mathcal{O}\left(\frac{1}{\left(-k \tau_{\text {mat }}\right)^{3}}\right),
$$

which doesn't make much difference for the estimation.

${ }^{4}$ We also observed that when the gCS term is replaced by $W \widetilde{W}$, in which $W$ is the Weyl tensor, the chirality oscillation also appears. The result is similar.
} 
difference of the amplitudes of both modes, which leads to the nearly opposite oscillating shapes in the left- and right-handed GWs spectrum.

The chirality oscillation of primordial GWs may bring obvious wiggles in the CMB TB/EB-mode spectrum, which is the unique fingerprint of chiral gravity. Thus highprecision CMB B-mode polarization experiments could offer us richer information on the UV-complete gravity theory than expected, though the detecting is still a challenging issue $[28,33,34]$.

\section{Acknowledgments}

This work is supported by NSFC, No. 11222546, 11575188, and the Strategic Priority Research Program of Chinese Academy of Sciences, No. XDA04000000.

Open Access. This article is distributed under the terms of the Creative Commons Attribution License (CC-BY 4.0), which permits any use, distribution and reproduction in any medium, provided the original author(s) and source are credited.

\section{References}

[1] L. Álvarez-Gaumé and E. Witten, Gravitational anomalies, Nucl. Phys. B 234 (1984) 269 [INSPIRE].

[2] R. Jackiw and S.Y. Pi, Chern-Simons modification of general relativity, Phys. Rev. D 68 (2003) 104012 [gr-qc/0308071] [inSPIRE].

[3] S. Alexander and N. Yunes, Chern-Simons Modified General Relativity, Phys. Rept. 480 (2009) 1 [arXiv:0907.2562] [InSPIRE].

[4] S. Weinberg, Effective Field Theory for Inflation, Phys. Rev. D 77 (2008) 123541 [arXiv:0804.4291] [INSPIRE].

[5] J.M. Maldacena and G.L. Pimentel, On graviton non-Gaussianities during inflation, JHEP 09 (2011) 045 [arXiv: 1104.2846] [INSPIRE].

[6] C.R. Contaldi, J. Magueijo and L. Smolin, Anomalous CMB polarization and gravitational chirality, Phys. Rev. Lett. 101 (2008) 141101 [arXiv:0806.3082] [INSPIRE].

[7] T. Takahashi and J. Soda, Chiral Primordial Gravitational Waves from a Lifshitz Point, Phys. Rev. Lett. 102 (2009) 231301 [arXiv:0904.0554] [INSPIRE].

[8] A.A. Starobinsky, Spectrum of relict gravitational radiation and the early state of the universe, JETP Lett. 30 (1979) 682 [INSPIRE].

[9] V.A. Rubakov, M.V. Sazhin and A.V. Veryaskin, Graviton Creation in the Inflationary Universe and the Grand Unification Scale, Phys. Lett. B 115 (1982) 189 [InSPIRE].

[10] Y. Cai, Y.-T. Wang and Y.-S. Piao, Oscillating modulation to B-mode polarization from varying propagating speed of primordial gravitational waves, Phys. Rev. D 91 (2015) 103001 [arXiv: 1501.06345] [INSPIRE].

[11] Y. Cai, Y.-T. Wang and Y.-S. Piao, Oscillation in power spectrum of primordial gravitational wave as a signature of higher-order stringy corrections, JHEP 02 (2016) 059 [arXiv: 1508.07114] [INSPIRE]. 
[12] D. Baumann, H. Lee and G.L. Pimentel, High-scale inflation and the tensor tilt, JHEP 01 (2016) 101 [arXiv: 1507.07250] [INSPIRE].

[13] A. Lue, L.-M. Wang and M. Kamionkowski, Cosmological signature of new parity violating interactions, Phys. Rev. Lett. 83 (1999) 1506 [astro-ph/9812088] [INSPIRE].

[14] N. Barnaby and M. Peloso, Large NonGaussianity in Axion Inflation, Phys. Rev. Lett. 106 (2011) 181301 [arXiv:1011.1500] [InSPIRE].

[15] L. Sorbo, Parity violation in the Cosmic Microwave Background from a pseudoscalar inflaton, JCAP 06 (2011) 003 [arXiv:1101.1525] [INSPIRE].

[16] P. Adshead, E. Martinec and M. Wyman, Gauge fields and inflation: Chiral gravitational waves, fluctuations and the Lyth bound, Phys. Rev. D 88 (2013) 021302 [arXiv:1301.2598] [INSPIRE].

[17] P. Adshead, E. Martinec and M. Wyman, Perturbations in Chromo-Natural Inflation, JHEP 09 (2013) 087 [arXiv: 1305.2930] [INSPIRE].

[18] R. Namba, M. Peloso, M. Shiraishi, L. Sorbo and C. Unal, Scale-dependent gravitational waves from a rolling axion, JCAP 01 (2016) 041 [arXiv: 1509.07521] [INSPIRE].

[19] M. Peloso, L. Sorbo and C. Unal, Rolling axions during inflation: perturbativity and signatures, JCAP 09 (2016) 001 [arXiv: 1606.00459] [INSPIRE].

[20] I. Obata and J. Soda, Oscillating Chiral Tensor Spectrum from Axionic Inflation, Phys. Rev. D 94 (2016) 044062 [arXiv: 1607.01847] [InSPIRE].

[21] Y. Cai, Y.-T. Wang and Y.-S. Piao, Is there an effect of a nontrivial $c_{T}$ during inflation?, Phys. Rev. D 93 (2016) 063005 [arXiv: 1510.08716] [INSPIRE].

[22] Y. Cai, Y.-T. Wang and Y.-S. Piao, Propagating speed of primordial gravitational waves and inflation, Phys. Rev. D 94 (2016) 043002 [arXiv: 1602.05431] [INSPIRE].

[23] S. Alexander and J. Martin, Birefringent gravitational waves and the consistency check of inflation, Phys. Rev. D 71 (2005) 063526 [hep-th/0410230] [INSPIRE].

[24] D.H. Lyth, C. Quimbay and Y. Rodriguez, Leptogenesis and tensor polarisation from a gravitational Chern-Simons term, JHEP 03 (2005) 016 [hep-th/0501153] [INSPIRE].

[25] M. Li, Y.-F. Cai, X. Wang and X. Zhang, CPT Violating Electrodynamics and Chern-Simons Modified Gravity, Phys. Lett. B 680 (2009) 118 [arXiv:0907.5159] [INSPIRE].

[26] Y.-T. Wang and Y.-S. Piao, Parity violation in pre-inflationary bounce, Phys. Lett. B 741 (2015) 55 [arXiv: 1409.7153] [INSPIRE].

[27] S. Saito, K. Ichiki and A. Taruya, Probing polarization states of primordial gravitational waves with CMB anisotropies, JCAP 09 (2007) 002 [arXiv:0705.3701] [INSPIRE].

[28] V. Gluscevic and M. Kamionkowski, Testing Parity-Violating Mechanisms with Cosmic Microwave Background Experiments, Phys. Rev. D 81 (2010) 123529 [arXiv:1002.1308] [INSPIRE].

[29] A. Ferté and J. Grain, Detecting chiral gravity with the pure pseudospectrum reconstruction of the cosmic microwave background polarized anisotropies, Phys. Rev. D 89 (2014) 103516 [arXiv: 1404.6660] [INSPIRE].

[30] M. Satoh and J. Soda, Higher curvature corrections to primordial fluctuations in slow-roll inflation, JCAP 09 (2008) 019 [arXiv:0806.4594] [INSPIRE]. 
[31] S. Dyda, E.E. Flanagan and M. Kamionkowski, Vacuum Instability in Chern-Simons Gravity, Phys. Rev. D 86 (2012) 124031 [arXiv:1208.4871] [INSPIRE].

[32] R.R. Caldwell, C. Devulder and N.A. Maksimova, Gravitational wave-gauge field oscillations, Phys. Rev. D 94 (2016) 063005 [arXiv: 1604.08939] [INSPIRE].

[33] W. Zhao and M. Li, Fluctuations of cosmological birefringence and the effect on CMB B-mode polarization, Phys. Rev. D 89 (2014) 103518 [arXiv:1403.3997] [InSPIRE].

[34] M. Gerbino, A. Gruppuso, P. Natoli, M. Shiraishi and A. Melchiorri, Testing chirality of primordial gravitational waves with Planck and future CMB data: no hope from angular power spectra, JCAP 07 (2016) 044 [arXiv: 1605.09357] [INSPIRE]. 